\title{
Editorial
}

\section{The Role of Obstetrician Gynecologist in Ovarian Cancer Management}

\section{Laila Nuranna}

Ovarian cancer is the second most common of female reproductive cancer in the world, more women die from ovarian cancer than from cervical cancer and uterine cancer combined. ${ }^{1}$ The role of screening in cervical cancer is prominent, but not in ovarian cancer. Currently, there is no strategy for early detection of ovarian cancer that reduces ovarian cancer mortality.

However, some effort should be done, to have better survival in the management of ovarian cancer, from taking a detailed family history for breast, gynecologic, and colon cancer facilitates categorizing women based on average high risk of developing ovarian cancer. Women with a strong family history of ovarian, breast, or colon cancer may have hereditary breast and ovarian cancer syndrome (BRCA mutation) or hereditary nonpolyposis colorectal cancer (Lynch syndrome). ${ }^{2}$ Ideally, women with these conditions should be offered for genetic counselling and additional testing for early detection of ovarian cancer. The use of transvaginal ultrasonography and tumour markers such as Ca 125 for the early detection of ovarian cancer have a role but have not been proved to reduce mortality. ${ }^{3}$

The new classification of histopathological based on molecular, and genetic studies have recently provided a better model for ovarian carcinogenesis, which are designated as Type I tumours include low grade serous, mucinous, endometrioid, clear cell, and transitional cell carcinomas, while type II tumours comprise high-grade serous carcinomas, undifferentiated carcinomas, and carcinosarcomas. ${ }^{4}$

It is very important to predict whether the adnexa tumour is malignant, borderline or benign pra operatively. Some scoring was implemented to predict the risk of malignancy of the adnexa tumour. We can use RMI (Risk Malignancy Index), Gatot Score, ROMA (Risk of Malignancy Algorithm), IOTA (International Ovarian Tumor Analysis). ${ }^{5,6}$ and also another scoring. We can have the application for that scoring formula.

Standard treatment for ovarian cancer involves aggressive debulking surgery and chemotherapy. Cytoreductive surgery aims to confirm the diagnosis, define the extent of disease, resect all visible tumour with the goal is zero residual tumours. Surgery in a case with early-stage can be done by ObGyn, with an understanding of the principles of surgery in ovarian cancer, including understanding the role of frozen section. But in advance stage, ideally managed by a team approach, consist of a gynaecologist, digestive surgeon, some times with the urology surgeon. Neoadjuvant chemotherapy is increasingly used. ${ }^{7}$ How to achieve better survival for ovarian cancer is challenging. 


\section{REFERENCES}

1. Siegel RL, Miller KD, Jemal A. Cancer statistics, 2016. CA Cancer. J Clin. 2016;66:7-30.

2. Family history as a risk assessment tool. Committee Opinion No. 478. American College of Obstetricians and Gynecologists. Obstet Gynecol. 2011;117:747-50.

3. The American College of Obstetricians and Gynecologists. The role of the obstetrician-gynaecologist in the early detection of epithelial ovarian cancer in women at average risk. Committee Opinion No. 716. American College of Obstetricians and Gynecologists. Obstet Gynecol 2017;130:e146-9.

4. Masafumi Koshiyama, Noriomi Matsumura, and Ikuo Konishi. Recent Concepts of Ovarian Carcinogenesis: Type I and Type II. Hindawi Publishing Corporation BioMed Research International 2014, Article ID 934261, 11 pages http://dx.doi.org/10.1155/2014/934261.

5. Bristow RE, Smith A, Zhang Z, Chan DW, Crutcher G, Fung ET, et al. Ovarian malignancy risk stratification of the adnexal mass using a multivariate index assay. Gynecol Oncol. 2013;128:252-9.

6. Sugandha Gard, Amarjit Kaur, Jaswandir Kaur Mohi, Preet Kanwal Sibia, Navkiran Kaur. Evaluation of IOTA Simple Ultrasound Rules to Distinguish Benign and Malignant. J Clin Diagn Res. 2017 ; 11(8): TC06-TC09. doi: 10.7860/ JCDR/2017/26790.10353.

7. Wright AA, Bohlke K, Armstrong DK, Bookman MA, Cliby WA, Coleman RL, et al,. Neoadjuvant Chemotherapy for Newly Diagnosed, Advanced Ovarian Cancer: Society of Gynecologic Oncology and American Society of Clinical Oncology Clinical Practice Guideline. J Clin Oncol. 2016;34(28):3460. 